\title{
Aprender a vivir con un órgano trasplantado
}

\author{
Learning to live with a transplanted organ \\ Aprendendo a viver com um órgão transplantado \\ Claudia Andrea Ramírez-Perdomo*
}

\section{Resumen}

Introducción: La insuficiencia Renal Crónica es una enfermedad silenciosa, cuando los síntomas aparecen la persona debe ingresar a una Terapia dialítica y/o iniciar el proceso del trasplante renal. El trasplante produce una mejoría en la calidad de vida de las personas enfermas. Objetivo: Analizar de manera comprensiva la experiencia vivida por personas con Insuficiencia Renal Crónica que han sido trasplantadas y los significados del cuidado de la salud elaborados por los familiares que interactúan con estas personas. Método: Estudio cualitativo, fenomenológico-hermenéutico. Se empleó el referente teórico de Van Manen. 11 personas enfermas y 5 cuidadores participaron en el estudio, en la ciudad de Neiva. La información fue recolectada a través de entrevistas en profundidad. Resultados: Al realizar el análisis emergió el tema "la esperanza de una segunda oportunidad, aprender a vivir con un órgano trasplantado", construido alrededor de los siguientes subtemas compartidos entre las personas enfermas y los cuidadores: la donación como un regalo de Dios, la llamada que anuncia el trasplante, reconocer la finitud del órgano y volver a nacer con el trasplante. Conclusiones: La donación es un obsequio que se espera pacientemente en el tiempo para poner fin a un tratamiento que produce miedo y desesperación. Esta alternativa de tratamiento lleva a los pacientes a volver a nacer y a recuperar la vida que han perdido por el tratamiento dialítico, aunque reconozcan que este es finito.

Palabras claves: Trasplante de riñón, insuficiencia renal crónica, hermenéutica, investigación cualitativa

\section{Abstract}

Introduction: Chronic kidney disease is an asymptomatic disease; when symptoms appear, the person has to receive dialysis and/or initiate the process of kidney transplantation. This transplant allows a better life quality of people with this disease. Objective: Analyze, in a comprehensive way, the experience of people with chronic kidney disease that have a transplant and the significance of the health care provided by the family members that interact with them. Method: Qualitative, hermeneutic phenomenological study, using the theoretical method of Van Manen, where 11 people with the disease and 5 caregivers participated in the study, made in the city on
Autor de correspondencia

* Enfermera, Magister en Enfermería. Doctora en Enfermería. Docente. Facultad de Salud, Universidad Surcolombiana. Correo: clauram1@hotmail.com. Orcid: http://orcid. org/0000-0001-9828-773X. Neiva, Colombia. 
ISSN-PRINT

1794-9831

E-ISSN 2322-7028

Vol. 16 No. 3

Sep - Dic 2019

Cúcuta, Colombia

Neiva-Colombia, with information recollected from in-depth interviews. Results: When doing the analysis the subject "hopes for a second opportunity, learning to live with an organ transplant" emerged, built around the following subcategories shared between people with the disease and the caregivers: donation as a gift of God, the call that anounces the transplant, recognizing the finitude of the organ and being reborn with the trasnplant. Conclusions: Donation if a gift that is waited on patiently through time, to put an end to a treatment that generates fear and desperation. This treatment alternative allows patients to be reborn and get back the life they have lost due to dialysis, although recognizing that this is finite.

Key words: Kidney transplantation, renal insufficiency, chronic, hermeneutics, qualitative research

\begin{abstract}
Resumo
Introdução: A insuficiência renal crônica é uma doença silenciosa; quando os sintomas aparecem, a pessoa precisa iniciar a terapia de diálise ou começar o processo para o transplante renal. Este transplante gera melhora na qualidade de vida destas pessoas. Objetivo: Analisar de maneira compreensiva a experiência vivida pelas pessoas com insuficiência renal crônica que tem sido transplantadas e os significados para a sua saúde que a família próxima percebe. Método: Estudo qualitativo, fenomenológico-hermenêutico empregando o referente teórico de Van Manen. Estudaram-se 11 pessoas doentes e 5 cuidadores moradores da cidade de Neiva. A informação coletou-se empregando a entrevista a profundidade. Resultados: Na análise surgiu o tema "a esperança de uma segunda oportunidade, aprender a viver com um órgão transplantado", construído pelos subtemas (identificados nos pacientes e cuidadores): a doação como um presente de Deus, a ligação anunciando o transplante, reconhecer o finito do órgão e voltar a nascer com o transplante. Conclusões: A doação é um presente que é esperado pacientemente, com a finalidade de encerrar um tratamento que gera medo e desesperação. Esta alternativa de tratamento leva aos pacientes a terem a sensação de renascença e de recuperação da vida que foi perdida pela diálise, embora reconheçam que é finito.
\end{abstract}

Palavras-chave: Transplante de rim, insuficiência renal, pesquisa qualitativa.

\section{Introducción}

La Insuficiencia Renal Crónica (IRC) es una enfermedad silenciosa, catastrófica, de evolución lenta, cuyo progreso puede tomar años y las manifestaciones clínicas aparecen cuando la pérdida de la función del riñón está por encima del 70\%; en algunos casos inclusive, con una función renal residual inferior a $20 \%$, los signos y síntomas no son evidentes (1). Las principales opciones de tratamiento son: el trasplante renal o la diálisis. Dadas las limitaciones de los trasplantes renales se reconoce que la gran mayoría de las personas ingresan a una Terapia de Reemplazo Renal (2).

El trasplante renal es el tratamiento ideal para las personas con IRC $(3,4)$ que mejora la calidad de vida y disminuye la mortalidad en la mayoría de los pacientes. En el discurso popular, el trasplante es presentado como "una cura" (5) de la enfermedad. La hemodiálisis (HD) es una terapia con mayor costo que el trasplante renal, por consiguiente es una alternativa que llevaría a reducir los costos en el tratamiento de las personas enfermas (6). Según Purnell et al. (7) la he- modiálisis mejora el funcionamiento físico, hay mayor participación social y de actividades recreativas, así como una mejor capacidad de trabajar en comparación con las personas que se encuentran en diálisis.

Por consiguiente, las personas con IRC desde el inicio del tratamiento deben ser informadas sobre el trasplante, que puede ser de donante vivo (familiar relacionado u otro compatible) o donante cadavérico. Con este tratamiento el paciente y la familia ponen todas sus esperanzas, porque significa para ellos salvar y mejorar su calidad de vida.

Adicionalmente a lo expuesto, es preciso señalar que pese a las mejoras logradas en el tratamiento con inmunosupresores (4), la vida del injerto trasplantado suele ser finita (8). El promedio de duración del injerto fluctúa y puede ir desde uno hasta diez años, con la tendencia a reducir el porcentaje de vida del riñón donado con el discurrir de los años.

La IRC y su tratamiento, es una realidad que produce cambios en la estructura y la dinámica de las relaciones interpersonales, donde la intersubjetividad que 
configura el mundo de la vida se fractura. El mundo de la vida es un tejido de significaciones que se construye y reconstruye permanentemente mediante las relaciones interpersonales, la experiencia y la comunicación (9). Así, la persona enferma y sus allegados sufren el embate de la enfermedad y su tratamiento, como una connotación social y el relacionarse no es ahora con la entidad nosológica, sino con el individuo, su familia y el impacto que se produce dentro de su cotidianidad.

Lo anteriormente descrito llevó a plantear como objetivo, analizar de manera comprensiva la experiencia vivida por personas con IRC que han sido trasplantadas y los significados elaborados por los familiares que interactúan con estos pacientes.

\section{Materiales y métodos}

Estudio cualitativo, fenomenológico y hermenéutico. Coherente con esa perspectiva, se busca mediante una indagación cara a cara reconstruir el significado de la experiencia vivida por las personas con IRC. Se explora el entorno socio-personal para comprender más profundamente su experiencia y derivar del conocimiento obtenido, un conjunto de lineamientos que permitan mejorar el cuidado a las personas que se encuentran en esta condición de salud.

El análisis se inició desde el momento de la transcripción de la entrevista siguiendo el método de Van Manen, en seis pasos: 1) recolección de la experiencia vivida, 2) reflexión de la experiencia vivida, 3) lectura y relectura de las entrevistas, 4) recuperar el lenguaje semántico dentro del texto, 5) detección de temas emergentes, 6) escritura y re-escritura de la experiencia vivida $(10,11)$.

La muestra se configuró con 11 participantes con IRC trasplantados y 5 cuidadores residentes en la ciudad de Neiva, Colombia. Fueron seleccionados mediante muestreo casual considerado como el más formal de la investigación cualitativa. Su lógica consiste en revisar y estudiar todos los casos que cumplan con los criterios predeterminados, asegurando la calidad de los datos (12). A través de este proceso los participantes brindaron información representativa (12). Es no probabilístico porque no se emplearon métodos aleatorios para la selección; estuvo orientado por criterio y escogido de la base de datos proporcionada por la unidad de trasplante Surcolombiano. Se tuvieron en cuenta los siguientes criterios de inclusión: personas con IRC trasplantadas mayores de 18 años, con trasplante renal por primera vez en un período mayor a seis meses, re-trasplantado en un período mayor a seis meses. Los cuidadores familiares debían ser mayores de 18 años y cuidador familiar principal. A cada uno se le asignó un seudónimo, para lo cual se decidió colocar las iniciales de los nombres; con el objetivo de diferenciar los grupos se colocó P (persona con IRC) y C (cuidador).

La información fue recolectada entre diciembre de 2015 a enero de 2017. Como técnica de recolección de la información se utilizó la entrevista en profundidad, donde se "aspira obtener el significado de la experiencia vivida" (10). Los participantes fueron contactados vía telefónica y entrevistados en su domicilio. Se partió de una pregunta general (13) que les permitiera hablar de manera amplia y establecer una conversación. Se presentó como pregunta de apertura al paciente: ¿Cuénteme su experiencia con el trasplante renal?; para los cuidadores familiares: ¿Cómo ha sido su experiencia con el trasplante renal de su familiar?. Adicionalmente se elaboró un listado de preguntas que permitieran ahondar en el fenómeno. Se realizaron 16 entrevistas en profundidad, una a cada una de las personas trasplantadas y a los cuidadores. Las entrevistas tuvieron una duración de una hora a hora y treinta minutos; fueron grabadas previo consentimiento del participante, después se escucharon y se transcribieron de manera textual por la investigadora.

Este proyecto estuvo avalado por el Comité de Ética de la Universidad de Antioquía, Acta No CEI-FE 2015-05. Se tuvieron en cuenta la Resolución 8430 de 1993 y los principios éticos de beneficencia, autonomía, privacidad, libertad de expresión y sentimientos. En el caso de la investigación cualitativa el investigador construye, recolecta, selecciona e interpreta datos. Se trata de un proceso de reflexividad que tiene un papel importante en este enfoque; el proceso de conexión dentro de la investigación parte de la introspección (14), sustentada en las experiencias personales, profesionales, los conocimientos y el interés por el fenómeno de estudio.

Se tuvieron en cuenta los criterios de rigor de Lincoln y Guba (15): credibilidad y confirmabilidad mediante la transcripción textual de las entrevistas. Los resultados fueron revisados por expertos en investigación cualitativa y devueltos a los participantes. Mediante 
ISSN-PRINT

1794-9831

E-ISSN 2322-7028

Vol. 16 No. 3

Sep - Dic 2019

Cúcuta, Colombia la transferibilidad se espera que los resultados sirvan para encontrar similitudes o divergencias con hallazgos de otras investigaciones que aborden este fenómeno.

\section{Resultados}

Las personas con IRC fueron seis mujeres y cinco hombres, con edad promedio de 45 años (DS $\pm 17,06$ ), nivel de escolaridad variada, tiempo promedio de trasplantado de 8,18 años. Los cuidadores familiares de las personas trasplantadas fueron cuatro mujeres y un hombre, con edad promedio de 45 años ( $\mathrm{DS}_{\underline{-}+5}, 3$ ), nivel de escolaridad diverso; la relación con las personas con IRC es el de esposa (o), madre y con ocupación diversa.

\section{La experiencia en el grupo de personas con IRC trasplantadas fue descrita de la siguiente manera:}

Para los pacientes la donación es un regalo de Dios. Al recibir el riñón donado se sienten bendecidos y agradecidos con la vida; igualmente, reconocen que su familia, aún a pesar del sufrimiento les brinda un regalo que les cambiará la existencia. De igual forma, toman la decisión de rechazar la donación del riñón de algún familiar por el temor de que ellos se vean afectados, esto lo sustentan en experiencias previas vividas por otras personas, en las cuales el donante y el donador terminan en tratamiento dialítico. Conseguir un donante es ganarse una lotería, es un regalo que se debe esperar pacientemente y mientras se espera, confiar en Dios como única certeza

Doy gracias a la familia que autorizó la donación y a ese angelito que está en el cielo, para ellos fue un gran dolor la pérdida de su ser querido. Mi mamá y mis hermanas se ofrecieron "nos hacemos los exámenes, tiene 5 para escoger", dije "No". En Bogotá vi el caso de una mamá que le donó a la hija, a los 10 años se le dañó el injerto a la hija y a los 12 años la mamá presentó falla renal y las dos iban a HD, entonces "dije una persona que no lo necesite". (PMCV).

Diosito me lo regaló. Hice el trámite del trasplante $y$ entré a lista de espera (...) El doctor me dijo que era una lotería y creo que me la gané (...). Me salió de donante cadavérico y no que mi hijo saliera de su riñón (...) (PMEA).

La llamada que anuncia el trasplante es un momento de temor y tensión. Estar preparado para el trasplan- te es importante, porque al recibir la llamada que lo anuncia, aparecen en el paciente los temores, que lo llevan a declinar la opción del trasplante; las experiencias previas negativas y los prejuicios, hacen que renuncie a este tratamiento. Sin embargo, conocer de cerca una experiencia positiva lo motiva a aceptar la opción de un donante cadavérico, y cuando llega la segunda llamada acude de inmediato al procedimiento, el cual termina siendo un éxito y se confirma con la presencia de la orina producida por el riñón. El instante en el que llega la llamada tan esperada, por regla general lo paraliza del miedo, se queda mudo. Apoyado por la familia oran, esperando que todo salga bien.

Al primer llamado no estaba preparada, tenía un compañero, a él no le fue bien en el trasplante, habia vuelto a diálisis y se quejaba "es que me colocaron un riñón que no servía, no estaba sano". Y tenía otros prejuicios "que se muera una persona sana para que me de el riñoncito, no es justo". En el segundo llamado ya estaba preparada. Me hicieron el trasplante, fue un éxito, a los tres días o cuatro días estaba en casa; oriné desde el momento en que me pusieron el riñoncito. (PMAA).

El día que me llamaron quede pasmada, no podia hablar, sentía el cuerpo helado de los nervios. Cuando me llamaron estaba mi mamá y mi familia, todo el mundo empezó a hacer oración, para que me fuera muy bien, los nervios fueron muy verracos (...) $(P V G)$.

Otro aspecto a tener en cuenta es el cumpleaños del trasplante, que es una forma de reconocer su finitud. Reconocer la finitud del trasplante produce temor. Ese cumpleaños es un tiempo regresivo para que deje de funcionar el riñón donado. No pensar en el tiempo en que dura el trasplante fortalece al paciente. Lo más importante es la obra que Dios hace para proteger su injerto hasta que llegue la muerte. Por otro lado, reconoce que también existe la opción de un nuevo trasplante o reingresar a tratamiento dialítico.

Hay temor, en estos días hablaba con una compañera y me dice "vamos a cumplir 8 años y faltan dos, el riñón cadavérico dura 10 años de vida", le dije "el mio está jovencito". El riñón dura hasta cuando Dios decide. La idea es morirme con este riñón, no quiero otro trasplante (...) (PMAA).

Al aparecer los síntomas del rechazo se inician con la batalla por comprender las causas que lo desarrolla- 
ron y las estrategias para prolongar un tiempo más la duración del trasplante. En esta lucha por conservar el trasplante no está contemplada la posibilidad de dejarse vencer por la adversidad, por el contrario, la meta es prolongar la vida del injerto.

Yo voy a cumplir cuatro años con el trasplante y este dura más o menos 5 años. Todos los problemas que he tenido antes ha durado (...). Los rechazos comenzaron el año pasado con el chicunguya, el organismo. Se hicieron todos estos exámenes de citomegalovirus, polyomavirus, los dos PRA, el cualitativo y el cuantitativo, cuando hicieron la biopsia se dieron cuenta que era una glomerulonefritis, era dificil conservar el injerto. Hemos intentado con las plasmaféresis para tratar de salvarlo. Estamos luchando para prolongarlo otros días, no me voy a dar por vencido, que se dañe el injerto y para diálisis (...) $(P E P)$.

Volver a nacer con el trasplante. Despiertan de la cirugía llenos de cables y lo describen como un momento doloroso. El instante crítico es después de la valoración cuando lo encuentran normal; empiezan a deambular. El levantarse de la cama es atemorizante, con unos cuantos cables atraviesan el pasillo que los lleva hacia la recuperación; ese espacio es la confirmación del cambio de vida. El poder orinar es la demostración de la función del nuevo riñón. Con el trasplante se vuelve a orinar, a comer y respirar mejor. Igualmente, se recupera la libertad, pueden desplazarse y vuelven a vivir la vida como antes, sin limitaciones, ni horarios; una vida en la cual no están atados a una máquina o a la enfermedad. Reconocen un cambio en su vida del cielo a la tierra, vuelven a nacer. Aunque continúe latente la enfermedad, ellos sienten que pueden volver a ser lo que eran antes y eso les produce una inmensa alegría.

Al otro día me dolía todo, cables por todas partes, sondas, drenes. Me llevaron a la habitación; llego el médico, me tomaron signos, y me dijo ¿Ve ese pasillo allá? le dije "Si señor", "Levántese vaya y venga", le dije "me acaba de sacar de cirugía", me levanté, fui y volví, al otro día me dieron salida. Uno vuelve a orinar, comer, respirar, se siente libre de poder desplazarse a muchas partes. Volver a vivir, a tener esa libertad de hacer lo que uno desea, sin tener que conectarse a una máquina (...) (PEYS).

Eso me ha permitido estar cerca de la gente que amo, volver a hacer cosas, estoy tomando clases de piano, dando clases. Es volver a recuperar lo que uno es, aunque uno no está el 100\% está en un 99\% y es una gran felicidad (...) (PMCV).

\section{La experiencia de los cuidadores se describe a continuación}

La donación es vista por los cuidadores como un regalo de Dios. Uno de los cuidadores que se convirtió en donante de su hijo considera que asumir la responsabilidad de ser el donante, lo llena de felicidad. Lo más importante es contribuir a que su familiar siga adelante, no le da miedo arriesgarse a un procedimiento complicado, dado que su hija tendrá una mejor calidad de vida al resultar exitoso el trasplante.

En el momento en que nos dijeron que podía servir para el trasplante, me puse contenta, tenía fe en Dios, que la niña iba a seguir bien. Nunca pensé que me iba a morir, dije "mi niña para adelante" (...). Me dijeron mañana es la cirugía, ni siquiera miedo me dio, estaba contenta de quitarme esa angustia de la enfermedad de la niña (...) (CIN).

Para ellos el trasplante es una bendición de Dios; tienen miedo, pero este se disipa al ver como cambia la vida de la persona enferma. Agradecen las bendiciones que han recibido: lograr la pensión, el trasplante y mejorar la calidad de vida como pareja. Crea sentimientos de arraigo en una ciudad que les ha brindado mucha prosperidad en su vida.

La espera fue corta, una lotería, una bendición. Teníamos miedo porque no sabíamos que iba a pasar, pero el cambio que ha tenido $C$ (...). Tenemos mejor calidad de vida, podemos ahorrar, cada año estamos viajando, podemos darnos ciertos lujos, nos ha ido muy bien y pues gracias a Dios acá tuvo su riñón (CSBR).

El cuidador comparte el temor al rechazo del trasplante y la pérdida del riñón con la persona enferma. Con frecuencia las personas enfermas plantean como alternativa no continuar con el tratamiento. Quien cuida se angustia ante el panorama de regresar a diálisis, ninguno de los dos desea volver a vivir esa experiencia.

Ella dice que era mejor que no le hubieran hecho nada, que si se le vuelve a dañar el riñón no vuelve a diálisis ni a trasplantarse. Desde hace 4 años el doctor le dijo "Dentro de 3 o 4 meses hay que colocarla en 
ISSN-PRINT

1794-9831

E-ISSN 2322-7028

Vol. 16 No. 3

Sep - Dic 2019

Cúcuta, Colombia lista de espera o usted vuelve a diálisis", para ella fue terrible y para mi también, no quiero volver a vivir esa experiencia (...) (CIN).

Ante el temor del rechazo, el cuidador apoya al enfermo y empieza junto a él un proceso de preparación ante esta eventualidad. Sin embargo, ellos esperan que la duración del trasplante sea prolongada.

Siempre le he tratado de hacer lavado cerebral, alguna vez lloró porque le da miedo perder el riñón $y$ dice que no la vaya a dejar meter a HD. Igual toca prepararse para eso, hay que prepararse para llegar a eso o peritoneal, esperamos que nos dure mucho el riñón (...) (CSBR).

Los cuidadores comparten la experiencia de volver a nacer con el trasplante. Re-encontrarse con su hija después del trasplante es un momento de inmensa felicidad. Ver la "bolsa llena de orines" representa un nuevo inicio, sus riñones vuelven a funcionar, esto los lleva a vislumbrar el cambio en la vida del familiar enfermo.

Estaba contenta de poder mejorar la calidad de vida a la niña (...) al tercer día todavía estaba hospitalizada cuando la entraron, para mi fue una alegría grande y esa bolsa llena de orines, un cambio de vida de la tierra al cielo (...) (CIN).

El trasplante les trae paz y tranquilidad, descansan del estrés y la angustia del tratamiento. Duermen mejor. Sienten que vuelven a nacer junto con la persona que es sometida al trasplante. Mientras están en tratamiento sienten que no tienen vida, ésta mejora una vez se realiza el trasplante a la persona enferma y se asume con responsabilidad la vida.

He descansado, sobre todo con las comidas, a la hora que llegara, asi estuviera cansada, le hacia la comida. Me siento tranquila, tanto para ellos y para uno es una nueva vida después del trasplante. Ahora vivo una vida como de un rey, se que él es una persona juiciosa y tiene una vida muchisimo mejor (CANP).

\section{Discusión}

La donación es percibida como una bendición de Dios, sienten agradecimiento con las familias que aprobaron la donación del órgano. Durante esta etapa la fe, es fuente de esperanza, les ayuda a lidiar con la incertidumbre en el proceso del trasplante, brindándoles consuelo y paz. Diversos autores describen la fe como un pilar fundamental en la vida $(16,17)$ y una de las estrategias de afrontamiento más empleadas (18) mientras que se espera la donación. Por lo tanto, consideran a Dios como el encargado de proporcionar los órganos para la donación (19).

Recibir la donación del órgano es una forma de ser liberados de la dureza del tratamiento y de la máquina de diálisis a la cual se encuentran atados. El trasplante como un regalo recibido de otra persona, los lleva a asumir el reto de protegerlo y la única forma que tienen de prevenir el daño del riñón, es a través de la toma de los medicamentos (20) inmunosupresores ordenados.

Con frecuencia se rechazan los donantes vivos intrafamiliares por el temor que se afecten al perder este órgano. El temor a recibir el órgano de un donador vivo, en este caso el familiar, es asimilado como un intento de poner en peligro la salud del donante; se sienten egoístas por acortarles la vida, convirtiéndose en prioridad la protección de sus seres amados del riesgo que plantea la donación (21). Así mismo, los receptores de la donación se preocupan de ser culpados si el donante se enferma o lamentan el sacrificio si el injerto falla. Por último y no menos importante es la preocupación por las molestias que el donador pueda sufrir, tales como: dolor, cambios en la apariencia física, afectación en el trabajo y la vida social.

Por otra parte, el familiar que asume el reto de donar, lo hace con amor, con total desprendimiento, buscando brindar una mejoría en la calidad de vida de la persona amada. A pesar de las vicisitudes que se presentan en la espera, ofrecer la donación del riñón para su familiar, los hace sentir que la espera ha dado resultados. El éxito del trasplante lleva a reconocer la mejoría en la calidad de vida de las personas. El donante familiar eleva su estatus en la familia y la persona enferma, ganando admiración y respeto; la carga emocional del donante que es visto como "héroe", es significativa (22).

Cabe destacar, que la llamada que anuncia el trasplante, es un momento en que las personas sienten temor y se paralizan, a pesar de ser el anuncio de la llegada del tan esperado evento. Se puede renunciar a la primera opción por miedo. De igual manera, el anuncio produce emoción, ante lo cual acuden inmediatamente al llamado. Este acontecimiento vaticina que el presente esta por cambiar y que el futuro deparará mejores 
condiciones en la vida, dejando atrás el pasado que ha sido doloroso, lleno de angustia, de restricciones y en el que se ha tenido que enfrentar múltiples pérdidas. La llamada pone fin al sufrimiento que la enfermedad y el tratamiento han impuesto en su vida.

Se puede señalar, que por el trasplante se espera en el tiempo, pero no el tiempo cronológico que marca el reloj, sino el tiempo en que se vive la experiencia de la espera paciente en que suceda el trasplante, un tiempo lleno de significados. Retomando a Heidegger (23), es el tiempo de estar-a-la-espera, en el cual aprenden a estar atentos y preparados, para cumplir la cita con el futuro incierto que les depara este, y que llegará de manera inesperada y sin ser anunciado, lo que significa el fin del "sufrimiento" en el cual viven desde el momento de ser diagnosticados con IRC.

No obstante, la opción del trasplante es la esperanza para mejorar la calidad de vida. Es así como vivir la experiencia de enfrentar su propia mortalidad, lo que los lleva a tomar conciencia de que el trasplante ocurre solamente con la muerte de alguien más $(24,25)$. A la espera se le agrega el tiempo de tratamiento, incrementando el impacto negativo de la enfermedad en su cotidianidad. Cuando se espera por un donante cadavérico, ese tiempo se acompaña de una gran incertidumbre, que produce estrés y ansiedad (20).

Habitualmente, las personas trasplantadas son conscientes de la finitud del riñón, la cuenta regresiva que indica el cumpleaños final del órgano produce desasosiego. La duración del órgano es el interrogante continuo que permea la vida de los trasplantados. Por este motivo, identifican diversos factores que influyen en la duración como: la defensa del cuerpo contra agentes extraños, la aparición de nuevas enfermedades y la edad del donante cadavérico. El reto para las personas trasplantadas es lograr que le injerto les dure el mayor tiempo posible.

Se debe resaltar, que el trasplante renal se vive con incertidumbre (26) debido a la consciencia de que el órgano puede fallar en cualquier momento, el miedo a presentar otras enfermedades por la inmunosupresión, los sentimientos de ansiedad por los efectos secundarios de los medicamentos y sentimientos de tristeza como resultado de un donante que fallece para convertirse en donador.

Coherente con la finitud, está el miedo a perder el trasplante y ante eso la inminencia de regresar a la terapia de diálisis. Cuando se presentan los síntomas del rechazo, comienza la lucha para evitar el desenlace; sin duda, los pacientes son conscientes de que es una batalla que probablemente perderán y tendrán que regresar a la vida de restricciones que impone la terapia dialítica, situación que produce angustia y temor.

De igual forma, son conscientes de que ante el fracaso del trasplante deberán regresar al tratamiento dialítico, incrementándose el miedo que experimentan. Por este motivo, reconocen la importancia de la toma de medicamentos inmunosupresores para ayudar a proteger el injerto, aun cuando esto los haga vulnerables al cáncer $(20,27)$.

De modo similar, el cuidador comparte con la persona enferma el temor de la pérdida del injerto. Ante la amenaza de la pérdida los cuidadores sienten angustia, desean continuar alejados de la terapia de diálisis, experiencia que no desean volver a vivir. Por otro lado, el cuidador considera que si se produce el rechazo ellos deberán continuar apoyando a su ser amado.

De la misma manera, con el trasplante "vuelven a nacer". El despertar de la cirugía es un momento de miedo, han llegado a una nueva etapa de la vida. Así mismo, el levantarse y deambular produce temor. También, están el miedo a "la caída del riñón", el que estén con muchos cables con los que deben atravesar el pasillo, un camino que los conduce hacia la recuperación. Igualmente, la presencia de la orina es indicio que su vida cambió, y que por tal motivo respiran y comen mejor. Se liberan de las ataduras impuestas por la enfermedad y el tratamiento, hacen planes y realizan actividades que la enfermedad los obligó a suspender.

Es de hacer notar, que el trasplante renal se transforma en una visa para escapar de la "enfermedad" $(20,28)$; un escape relacionado con el hecho de liberarse del tratamiento de diálisis, así tengan que someterse a los factores de estrés propios de postrasplante. En esta etapa se "aferran" al éxito del trasplante, gracias a que les brinda un estilo de vida muy cercano a la "normalidad" experimentada antes de padecer la enfermedad. De ahí que asocien la vida en tratamiento dialítico como una vida con enfermedad, mientras que vivir con el trasplante del riñón a la vida de un individuo sano.

En consecuencia, el trasplante cobra el significado de renacer y comenzar una nueva vida. La presencia de 
ISSN-PRINT

1794-9831

E-ISSN 2322-7028

Vol. 16 No. 3

Sep - Dic 2019

Cúcuta, Colombia un nuevo órgano en su cuerpo, hace que experimenten alegría y felicidad; se sienten relajados, optimistas y pacientes para lidiar con los problemas de la vida $(19,29,30)$.

Por otra parte, la familia y/o sus cuidadores, comparten los sentimientos de alegría por el trasplante exitoso, descansan de la zozobra permanente en que han vivido, recuperan la paz y la tranquilidad, vuelven a hacer planes con sus seres queridos y reconocen que para ellos también la vida a cambiado. La donación los alivia de las restricciones y las cargas que se les impone, esta nueva situación da flexibilidad para viajar y socializar $(21,31)$.

Por tal motivo, las familias se readaptan a la nueva situación descrita como un momento de cambio. Esta etapa les permite hacer planes a corto, mediano y largo plazo; sienten que pueden cuidarse más, retornar al trabajo, teniendo en cuenta que antes del procedimiento la principal preocupación era dedicarse a cuidar a la persona enferma, incluso hasta descuidar su salud y la interacción social (22).

En conclusión, el volver a nacer les permite escapar del espacio donde se encontraban confinados por el dolor, el sufrimiento, la angustia y las restricciones impuestas por el tratamiento. A pesar de que la enfermedad permanece presente en sus vidas, con el trasplante los pacientes recuperan gran parte de la vida que tenían antes de ser diagnosticados y estar en tratamiento dialítico.

\section{Conclusiones}

- Para las personas trasplantadas, el tiempo de espera por la llamada que anuncia el trasplante, es una espera paciente y llena de angustia. Ese anuncio significa el fin de las ataduras a un tratamiento que genera sentimientos, desesperación y miedo.

- De igual manera, el trasplante es una experiencia compartida entre las personas trasplantadas y los cuidadores, asumiendo el significado de un regalo de Dios que debe ser agradecido y el cual genera sentimientos de reciprocidad. Es volver a nacer a una nueva vida, en la cual se recuperan parcialmente muchas de las cosas perdidas a raíz de la enfermedad y su tratamiento. Sin embargo, la enfermedad no desaparece con el trasplante, simplemente la vida les da una opción de poner fin, al menos de manera transitoria, al tratamiento de la diálisis. Un nuevo órgano se aloja en sus vidas como ese inquilino que se añora con alegría y felicidad. No obstante, el miedo al rechazo del órgano, la señal de la finitud de este, genera angustia en las personas trasplantas y sus cuidadores ante la inminencia de regresar al pasado, con un futuro incierto y un presente atemorizante.

\section{Conflicto de intereses}

Declaro que para el desarrollo de la presente investigación no existieron conflicto de intereses.

\section{Referencias Bibliográficas}

1. Gamarra G. Epidemiología de la insuficiencia renal crónica. Acta Medica Colomb. 2013;38(3):116-7.

2. Schaepe $C$, Bergjan M. Educational interventions in peritoneal dialysis: A narrative review of the literature. Int J Nurs Stud. 2015;52(4):882-98.

3. Perl J, Hasan $\mathrm{O}$, Bargman J, Jiang $\mathrm{D}, \mathrm{Na} Y$, Gill J, et al. Impact of dialysis modality on survival after kidney transplant failure. Clin J Am Soc Nephrol. 2011;6(3):582-90.

4. Ayar Y, Ersoy A, Ocakoglu G, Yildiz A, Oruc A, Soyak H, et al. Risk Factors Affecting Graft and Patient Survivals After Transplantation From Deceased Donors in a Developing Country: A SingleCenter Experience. Transplant Proc. 2017;49(2):270-7.

5. Boaz A, Morgan M. Working to establish "normality" post-transplant: A qualitative study of kidney transplant patients. Chronic Illn. 2014;10(4):247-58.

6. Rees M, Dunn T, Kuhr C, Marsh C, Rogers J, Rees S, et al. Kidney Exchange to Overcome Financial Barriers to Kidney Transplantation. Am J Transplant. 2017;17(3):782-90.

7. Purnell T, Auguste P, Crews D, Lamprea J, Olufade T, Greer R, et al. Comparison of Life Participation Activities Among Adults Treated by Hemodialysis, Peritoneal Dialysis, and Kidney Transplantation: 
A Systematic Review. Am J Kidney Dis. 2013;62(5):953-73.

8. Gill P, Lowes L. Renal transplant failure and disenfranchised grief: participants' experiences in the first year post-graft failure--a qualitative longitudinal study. Int J Nurs Stud. 2014;51(9):1271-80.

9. Schutz A, Luckmann T. Las estructuras del mundo de la vida. 1a ed. Amorrortu, editor. Buenos Aires; 1973. $320 \mathrm{p}$

10. Van Manen M. Investigacion educativa y experiencia vivida: ciencia humana para una pedagogía de la acción y la sensibilidad. Barcelona: Editorial Idea Books; 2003.

11. Van Manen M. Phenomenology of practice: meaning-giving methods in phenomenological research and writing. California: Editorial Left Coast Press; 2014.

12. Patton M. Qualitative evaluation methods and Research Methods. London: Editorial Sage; 2002.

13. Smith J. Qualitative psychology: a practical guide to research methods. London: Editorial Sage; 2008.

14. Finlay L. Negotiating the swamp: the opportunity and challenge of reflexivity in research practice. Qual Res. 2002;2(2):209-30.

15. Lincoln Y, Guba E. Naturalistic inquiry. California: Editorial Sage; 1985.

16. Davis L, Grogan T, Cox J, Weng F. Inter- and Intrapersonal Barriers to Living Donor Kidney Transplant among Black Recipients and Donors. J Racial Ethn Heal Disparities. 2017;4(4):671-9.

17. Sieverdes JC, Raynor PA, Armstrong T, Jenkins CH, Sox LR, Treiber FA. Attitudes and perceptions of patients on the kidney transplant waiting list toward mobile health-delivered physical activity programs. Prog Transplant. 2015;25(1):26-34.

18. Gurkan A, Pakyuz SÇ, Demir T. Stress Coping Strategies in Hemodialysis and Kidney Transplant Patients. 2015;47(5)1392-7.

19. Sheikhalipour Z, Zamanzadeh V, Borimnejad L, Valizadeh L, Shahbazi M, Zomorrodi A, et al. Recipients' experiences after organ transplantation. Int J Organ Transplant Med. 2018;9(2):88-96.

20. Low J, Crawford K, Manias E, Williams A. Stressors and coping resources of Australian kidney transplant recipients related to medication taking: a qualitative study. J Clin Nurs. 2017;26(11-12):1495507.

21. Hanson C, Chadban S, Chapman J, Craig J, Wong G, Ralph A, et al. The Expectations and Attitudes of Patients With Chronic Kidney Disease Toward Living Kidney Donor Transplantation: A Thematic Synthesis of Qualitative Studies. Transplantation. 2015;99(3):540-54.

22. Da Silva Cruz M, Daspett C, De Aguiar Roza B, Da Silva Ohara C, De Moraes Horta A. Family experience in the kidney transplant process from a living donor. Acta Paul Enferm. 2015;28(3):275-80.

23. Heidegger M. El ser y el tiempo. México: Editorial Fondo de Cultura Económica; 1974.

24. Burns T, Fernandez R, Stephens M. The experiences of adults who are on dialysis and waiting for a renal transplant from a deceased donor: a systematic review. JBI database Syst Rev Implement reports. 2015;13(2):169-211.

25. Schipper K, Abma TA, Koops C, Bakker I, Sanderman R, Schroevers MJ. Sweet and sour after renal transplantation: A qualitative study about the positive and negative consequences of renal transplantation. Br J Health Psychol. 2014;19(3):580-91.

26. Lonargáin $\mathrm{D}$, Brannigan $\mathrm{D}$, Murray $\mathrm{C}$. The experience of receiving a kidney transplant from a deceased donor: Implications for renal services. Psychol Heal. 2017;32(2):204-20.

27. Muduma G, Shupo FC, Dam S, Hawken NA, Aballéa S, Odeyemi I, et al. Patient survey to identify reasons for non-adherence and elicitation of quality of life concepts associated with immunosuppressant therapy in kidney transplant recipients. Patient Prefer Adherence. 2016;10:27-36.

28. Bailey PK, Ben-Shlomo Y, Tomson CR V, Owen-Smith A. Socioeconomic deprivation and barriers to 
ISSN-PRINT

1794-9831

E-ISSN 2322-7028

Vol. 16 No. 3

Sep - Dic 2019

Cúcuta, Colombia live-donor kidney transplantation: A qualitative study of deceased-donor kidney transplant recipients. BMJ Open. 2016;6(3): e010605.

29. Chatrung C, Sorajjakool S, Amnatsatsue K. Wellness and Religious Coping Among Thai Individuals Living with Chronic Kidney Disease in Southern California. J Relig Health. 2015;54(6):2198211.

30. Rao PS, Nunes JW. Renal transplant failure has a devastating impact requiring greater recognition and support. Evid Based Nurs. 2015;18(3):78.

31. Schmid Mohler G, Schäfer Keller P, Frei A, Fehr T, Spirig R. A Mixed-Method Study to Explore Patients' Perspective of Self-Management Tasks in the Early Phase after Kidney Transplant. Prog Transplant. 2014;24(1):8-18. 